\title{
O Desamparo Aprendido Revisitado: Estudos com Animais ${ }^{1}$
}

\author{
Maria Helena Leite Hunziker ${ }^{2}$ \\ Universidade de São Paulo
}

\begin{abstract}
RESUMO - O desamparo aprendido tem sido definido como a dificuldade de aprendizagem apresentada por indivíduos que tiveram experiência prévia com estímulos aversivos incontroláveis. O objetivo deste trabalho é fazer uma revisão crítica dos estudos sobre o desamparo aprendido, com animais. Nessa análise, são considerados aspectos conceituais e metodológicos dos estudos em questão e as interpretações teóricas sobre esse efeito comportamental. Aborda-se a evolução histórica desses estudos, bem como alguns aspectos controversos das publicações que se acumularam ao longo de quatro décadas de pesquisa. A associação do desamparo aprendido com a depressão clínica é analisada criticamente, destacando-se a necessidade de maior rigor metodológico e conceitual nos estudos da área.
\end{abstract}

Palavras-chave: desamparo aprendido; depressão experimental; controle aversivo; modelo animal de depressão.

\section{Learned Helplessness Revisited: Animal Studies}

\begin{abstract}
Learned helplessness is a learning deficit produced by the previous experience with uncontrollable aversive stimuli. The present paper aims at carrying out a critical review of studies on animal learned helplessness. Conceptual and methodological questions related to learned helplessness are considered, along with its theoretical interpretation. We analyze both the historical evolution of studies on learned helplessness and some controversial experimental results obtained during four decades of research. The relationship between learned helplessness and clinical depression is also discussed, with emphasis on the necessity for a rigorous methodological and conceptual analysis.
\end{abstract}

Key words: learned helplessness; experimental depression; aversive control; animal model of depression.

O desamparo aprendido tem sido definido como a dificuldade de aprendizagem apresentada por indivíduos que tiveram experiência prévia com estímulos incontroláveis (Maier \& Seligman, 1976). Segundo Peterson, Maier e Seligman (1993), a identificação do desamparo aprendido se deu de forma acidental. Os primeiros trabalhos foram realizados na década de 1960, quando a Teoria dos Dois Fatores estava no centro das discussões, sugerindo que o comportamento de esquiva decorreria de dois processos de aprendizagem: um processo respondente - produzido pelo pareamento do estímulo aversivo com o estímulo que o precedesse sistematicamente (transferindo a este a função de estímulo aversivo condicionado, ou CS) -, e outro processo operante, no qual o reforçamento seria decorrente do término do CS (Mowrer, 1947; Rescorla \& Solomon, 1967). Nessa época, Bruce Overmier e Russell Leaf, dois alunos de pós-graduação orientados por Solomon (um dos defensores dessa teoria), decidiram manipular a ordem de aquisição dos condicionamentos respondente e operante para verificar se isso interferiria na aquisição de esquiva. Para tanto, liberaram choques e estímulos sonoros em diferentes ordens de apresentação, pareados ou não, relacionados ou não com a resposta de esquiva. Dentre as várias manipulações efetuadas, eles expuseram um grupo de cães, presos em arreios, à associação luz/choque (associação CS/US). Como essa fase buscava propiciar apenas a aprendizagem respondente, nenhuma resposta

1 Esse trabalho é parte da dissertação de Livre-Docência, defendida na Universidade de São Paulo, em 2003. A autora agradece a leitura crítica de Cristiano Valério dos Santos e o apoio financeiro do CNPq .

2 Endereço: Universidade de São Paulo, Av. Prof. Mello Moraes, 1721, São Paulo, SP, Brasil 05508-900. E-mail: hunziker@lexxa.com.br desses cães produzia qualquer modificação nos choques, que eram, portanto, incontroláveis. Posteriormente, esses animais foram colocados em uma caixa onde um tom antecedia choques por um intervalo fixo de tempo: caso o sujeito saltasse para o compartimento oposto da caixa, o som era desligado e o choque evitado (contingência de esquiva). Ao longo desse teste, o CS luz (utilizado na primeira fase) era também apresentado, independentemente da contingência de esquiva em vigor, com a finalidade de verificar se esse CS teria se tornado aversivo a ponto de aumentar a probabilidade da resposta de saltar. Contrariando a teoria em avaliação, obtiveram que a luz não aumentou a probabilidade de saltar. Contudo, um resultado inesperado lhes chamou a atenção: apesar de estar vigorando uma contingência de reforçamento negativo, a resposta de esquiva não foi aprendida. Esses resultados, paralelos aos objetivos da pesquisa, sugeriram que choques incontroláveis podiam afetar novas aprendizagens operantes negativamente reforçadas (Overmier \& Leaf, 1965).

Em função desses resultados, Martin Seligman (outro pós-graduando daquele laboratório) se associou a Bruce Overmier, passando a investigar essa interferência da incontrolabilidade sobre a aprendizagem. Para isso, expuseram grupos de cães a choques incontroláveis ou a nenhum choque e, posteriormente, submeteram esses animais a uma contingência de reforçamento negativo. Na sua primeira publicação sobre o tema, Overmier e Seligman (1967) mostraram que os animais anteriormente submetidos a choques incontroláveis não aprenderam a resposta prevista no teste, ao contrário dos cães não submetidos aos choques, que aprenderam a resposta de fuga rapidamente.

Apesar de ser pioneiro nessa área, esse trabalho não separou experimentalmente os efeitos dos choques daqueles 
decorrentes da sua incontrolabilidade. Isso foi feito em seguida, tendo Seligman e Maier (1967) estudado simultaneamente três sujeitos, cada um pertencente a um grupo distinto, caracterizando o que se passou a chamar "delineamento triádico". Na primeira fase, dois cães foram submetidos simultaneamente a choques elétricos, enquanto o terceiro animal permanecia na caixa experimental, sem choques. $\mathrm{O}$ controle sobre os choques era permitido apenas a um dos cães que, submetido a uma contingência de fuga, podia desligá-los emitindo uma resposta previamente selecionada (pressionar um painel com o focinho). Quando isso ocorria, sua resposta também desligava o choque do segundo sujeito, cujas respostas não tinham conseqüência programada. Assim, apesar de ambos os cães receberem choques similares (no que diz respeito a número, intensidade, intervalo, etc.), apenas o primeiro podia exercer controle sobre esses estímulos; o segundo animal, por não poder alterar a duração dos choques, encontrava-se exposto à condição definida como de incontrolabilidade. Vinte e quatro horas depois, cada sujeito foi exposto a uma sessão na qual a resposta de correr era negativamente reforçada. Os resultados mostraram que tanto os animais previamente tratados com choques controláveis, como os não expostos a choques, aprenderam igualmente a resposta de fuga, mostrando latências gradual e sistematicamente mais baixas ao longo da sessão. Ao contrário, os animais previamente expostos aos choques incontroláveis não emitiram a resposta de fuga ou, quando o fizeram, não houve aumento subseqüente na probabilidade de ocorrência dessa resposta. Como resultado geral, esses sujeitos apresentaram latências altas durante toda a sessão; portanto, não aprenderam a resposta de fuga.

A essa dificuldade de aprender uma relação operante, decorrente da exposição prévia a eventos aversivos incontroláveis, deu-se o nome de efeito de interferência (Overmier \& Seligman, 1967; Seligman \& Maier, 1967). Apesar de essa denominação ser bastante descritiva do efeito comportamental em estudo, ela foi rapidamente substituída pelo termo "desamparo aprendido" (learned helplessness), o qual também denominou uma das hipóteses explicativas do fenômeno, conforme se verá mais à frente (Maier, Seligman \& Solomon, 1969; Maier \& Seligman, 1976). Por alguns anos, houve a tentativa de manutenção da terminologia original, isenta de conotação teórica (Crowell \& Anderson, 1981; Glazer \& Weiss, 1976a). Contudo, o termo desamparo aprendido foi o mais difundido, afirmando-se como denominação do efeito (Seligman, 1975; Peterson \& cols. 1993), apesar da indesejável simbiose que se estabeleceu entre o efeito e a sua interpretação teórica.

Algumas variações no procedimento básico possibilitaram a expansão da análise do desamparo aprendido. Dois procedimentos podem ser destacados, um voltado à "prevenção" e outro ao "tratamento" do desamparo. Para testar a "prevenção", foi analisada a importância da ordem de aprendizagem estabelecida. Considerando-se que o comportamento é um fenômeno cumulativo e que a exposição à incontrolabilidade promove a aprendizagem de que o estímulo independe da resposta, é de se esperar que uma primeira aprendizagem de controle sobre esse estímulo possa minimizar os efeitos de experiências futuras com a sua incontrolabilidade. Para verificar experimentalmente essa suposição, um grupo de cães foi submetido a uma primeira sessão com choques controláveis (fuga/esquiva), seguida por outra na qual eram expostos a choques incontroláveis. Verificou-se que, quando testados posteriormente em fuga/esquiva, esses animais aprenderam normalmente a resposta no teste. Esse efeito foi chamado de imunização, pois sugeria que o controle exercido na primeira sessão teria "imunizado" os sujeitos contra o aprendizado posterior da incontrolabilidade, deixando-os aptos a aprenderem a relação operante estabelecida no teste (Seligman \& Maier, 1967). Portanto, o estudo do efeito de imunização mostrou que a história de reforçamento pode ser uma variável crítica na prevenção contra o desamparo.

Haveria um procedimento que poderia reverter o desamparo, depois que esse padrão de comportamento já estivesse estabelecido? Para responder a essa questão, Seligman e Maier (1967) selecionaram cães que haviam apresentado desamparo e os re-expuseram à contingência de fuga do teste, forçando-os fisicamente a experimentar o reforçamento. Isso foi feito prendendo correias ao seu corpo e colocando os animais novamente na caixa de teste, que era dividida em dois compartimentos por uma pequena barreira. Ao iniciar o choque, os experimentadores puxavam os cães com as correias, na direção da barreira, até que eles a saltassem, indo para o compartimento oposto. Essa resposta de saltar era imediatamente seguida pelo término do choque. A cada choque, esse procedimento era reiniciado. Depois de expostos seguidamente a essa contingência de fuga forçada, os cães passaram a emitir a resposta de fuga sem a ajuda dos experimentadores, mostrando a reversão do desamparo. Com outro procedimento, buscou-se "tratar" os animais previamente expostos a choques incontroláveis expondo-os repetidamente a contingências de reforçamento positivo. Os estudos têm mostrado que, embora inicialmente os animais apresentem baixa taxa de respostas, o contato repetido com o reforçamento positivo reduz os efeitos da incontrolabilidade dos choques (Erbetta, 2004).

\section{Interpretações teóricas}

A análise teórica dos processos envolvidos no desamparo aprendido tomou duas direções opostas: uma que defende que, a despeito do arranjo experimental, o sujeito aprende a controlar o estímulo (por meio de contingências acidentais), e outra que propõe o aprendizado da impossibilidade de controle. A primeira interpretação embasa a hipótese da inatividade aprendida. Ela considera que o desamparo não é um efeito direto da incontrolabilidade dos choques, mas sim fruto de contingências acidentais, que se estabelecem de forma não programada pelo experimentador, e selecionando baixa atividade motora. Conseqüentemente, se o animal aprende a ficar pouco ativo, ele fica menos apto a emitir a resposta de fuga no teste, caso essa resposta envolva alta movimentação corporal (tal como correr, saltar ou pressionar a barra). Essa proposição considera, portanto, que a incontrolabilidade não atua diretamente sobre o comportamento, mas apenas estabelece a condição propícia para que surjam contingências acidentais. Uma possibilidade seria que a grande movimentação corporal eliciada pelos primeiros choques coincidiria com a continuidade dos mesmos, gerando uma punição da alta atividade motora (Bracewell \& Black, 1974). 
Outro processo acidental possível envolveria reforçamento negativo da inatividade. Ela foi sugerida pela observação de que a movimentação corporal tende a se reduzir após alguns segundos de choque, possibilitando que o término do choque coincida com baixa atividade motora (Glazer \& Weiss, 1976a, 1976b). Portanto, isoladamente ou em conjunto, essas duas contingências acidentais levariam à inatividade, gerando o desamparo aprendido.

Em direção contrária à anterior, a hipótese do desamparo aprendido pressupõe que o efeito obtido é fruto direto da impossibilidade de controle do ambiente: o sujeito aprende que não existe relação entre suas respostas e os estímulos, aprendizagem essa que se contrapõe à aprendizagem seguinte que envolve contingência de reforçamento (Maier \& cols., 1969; Maier \& Seligman, 1976).

Deve-se ressaltar que a "hipótese do desamparo aprendido" extrapola a análise das relações funcionais objetivamente estabelecidas na condição experimental e considera como críticos alguns processos cognitivos/mentalistas, inferidos a partir dos dados. Segundo seus proponentes (Maier \& cols., 1969; Maier \& Seligman, 1976), a variável independente crítica para o desamparo não é a incontrolabilidade estabelecida experimentalmente, mas sim a expectativa desenvolvida pelo indivíduo de que ele não pode controlar o ambiente. Essa expectativa atuaria em diferentes níveis, promovendo um conjunto de efeitos que comporiam o desamparo como uma síndrome, e não como um simples comportamento, que abarcaria três tipos de déficits: motivacional, cognitivo e emocional. O déficit motivacional seria, do ponto de vista descritivo, caracterizado pela baixa probabilidade da resposta no teste. No nível interpretativo cognitivista, é sugerido que após os choques incontroláveis o sujeito cria a "expectativa" de que o reforçamento não vem e por isso não tem motivo para emitir respostas no teste. Por sua vez, o déficit cognitivo é objetivamente caracterizado pelo fato do animal não ter seu comportamento modificado pelo reforçamento negativo: mesmo que o animal emita algumas respostas de fuga no teste e experimente que essa resposta é seguida pelo término do choque, seu comportamento não fica sob o controle dessa relação de conseqüenciação. Segundo a interpretação cognitivista desse efeito, ele decorre de uma alteração na forma como o sujeito processa a informação relativa à nova contingência. Seria esse "erro de processamento", causado pela "expectativa" de incontrolabilidade, que o levaria a não registrar a relação de dependência que há entre suas respostas e as mudanças no ambiente. Por fim, o déficit emocional é caracterizado por alterações fisiológicas, tais como mudanças do ciclo de sono e de ingestão de alimentos, imunossupressão, entre outras. Na interpretação cognitivista, a "crença" de que o reforço não virá, produz estados alterados de emoções (ansiedade e depressão) que, por sua vez, levam a essas alterações fisiológicas.

A hipótese do desamparo tem, portanto, dois níveis de apresentação que precisam ser analisados separadamente: (1) o nível descritivo, que diz respeito aos dados experimentais obtidos, e (2) o nível interpretativo, baseado em processos mentalistas que são inferidos dos dados experimentais. O nível descritivo é bem estabelecido cientificamente, permitindo previsão e controle dos comportamentos em estudo. As interpretações mentalistas não são aceitas por unanimidade, principalmente na perspectiva do behaviorismo radical (Skinner,
1974). Além dessa divergência filosófica, deve-se considerar que o caráter explicativo dado à suposta "expectativa" cria uma circularidade de análise bastante indesejável cientificamente: a dificuldade de aprendizagem é o dado que indica a "expectativa", e essa "expectativa" é a causa da dificuldade de aprendizagem (ou seja, diz-se que o sujeito apresenta o desamparo porque criou a expectativa de não ter controle sobre o meio, e sabe-se que ele criou essa expectativa porque apresentou desamparo). Com essas características, a hipótese do desamparo permite apenas explicações post hoc: só se pode deduzir o desenvolvimento da "expectativa" através da dificuldade de aprender e não pela exposição pura e simples à incontrolabilidade. Assim, não existe previsão de resultado antes de o trabalho ser realizado. Resultados negativos (aprendizagem mesmo após experiência com incontrolabilidade) podem ser explicados sem refutar a hipótese: se o sujeito não apresentou o desamparo é porque, apesar da experiência com incontrolabilidade, ele não desenvolveu a "expectativa" de impossibilidade de controle (ver análise dessa circularidade em Levis, 1976).

Apesar desses pontos criticáveis, a hipótese do desamparo tem a seu favor o fato de considerar que o comportamento é sensível à condição de incontrolabilidade, suposição essa compatível com a sensibilidade já identificada a pequenas variações no contínuo de dependência entre resposta $(\mathrm{R})$ e estímulo (S), descrita pelos analistas do comportamento (Catania, 1998). Do ponto de vista lógico, se há sensibilidade para variações no contínuo de controlabilidade (nas diferentes contingências operantes), deve haver também para a condição de incontrolabilidade. Assim, sob a perspectiva do behaviorismo radical, o expurgo do mentalismo na hipótese do desamparo aprendido pode torná-la útil como instrumento de análise dos resultados experimentais: a variável independente, incontrolabilidade dos choques, pode ser definida operacionalmente e, portanto, não depende de julgamentos subjetivos do experimentador; a emissão da resposta de fuga, em função do reforçamento negativo, é a variável dependente em estudo. Assim, têm-se em mãos as variáveis necessárias para se fazer a análise funcional dos comportamentos emitidos, envolvendo basicamente processos operantes e respondentes, sem ser necessário falar em motivação/incentivo/expectativa.

Essa releitura da hipótese do desamparo aprendido considera que o arranjo de incontrolabilidade não produz reforçamento diferencial de qualquer resposta. O que é aprendido (e supostamente generalizado para a condição futura) é justamente a ausência de relação R-S. Não havendo conseqüenciação sistemática, a alta movimentação corporal, eliciada pelos primeiros choques, tende a reduzir em função de um processo de habituação, ocorrendo de forma gradualmente menos intensa. Conseqüentemente, a freqüência e a intensidade da movimentação corporal caem ao longo da sessão, deixando o sujeito pouco ativo. Uma vez que o teste tem muitos estímulos comuns à fase de tratamento, é possível que, ao menos no início da sessão, essa passividade se generalize. Assim, pode-se prever que o sujeito se comporte no início do teste da mesma forma que vinha se comportando na fase anterior, ou seja, de forma pouco ativa. Porém, a mera inatividade não explica todo o processo, pois é freqüente que o animal emita a resposta de fuga, experimentando o término 
do choque contingente a ela, sem que isso aumente a probabilidade futura de emissão dessa resposta. De acordo com essa hipótese, a insensibilidade ao reforçamento negativo se deve à aprendizagem prévia de ausência de relação R-S, que é oposta à aprendizagem de fuga que envolve uma relação de dependência entre a resposta e o término do choque. Sendo opostas essas aprendizagens, é de se esperar que a primeira dificulte a seguinte, produzindo o desamparo.

Essa análise permite prever a baixa atividade geral que se observa ao final da sessão de choques incontroláveis, bem como o desamparo no teste, sem, contudo, vincular um efeito como causa do outro. O ponto crítico é que não há seleção operante na sessão de incontrolabilidade, condição oposta à do teste, que busca selecionar uma classe específica de respostas. Portanto, o procedimento empregado nos estudos sobre desamparo permite confrontar dois tipos de aprendizagens, relativas à incontrolabilidade e à controlabilidade do ambiente, sendo a sua ordem de ocorrência crítica para a produção dos efeitos de desamparo ou de imunização.

As hipóteses do desamparo aprendido e da inatividade aprendida oferecem previsões de efeitos contrários em decorrência de algumas manipulações, o que permite seu teste experimental. Diversos estudos foram realizados com esse fim, a maioria deles mostrando resultados contrários à hipótese da inatividade. Por exemplo, Yano e Hunziker (2000) realizaram dois experimentos para confrontar essas hipóteses, nos quais a variável manipulada foi o grau de atividade física requerido pela resposta de fuga no teste. Considerando o proposto pela hipótese motora, se o indivíduo aprende a ficar inativo durante os choques incontroláveis, então o desamparo só ocorre se a resposta de teste envolver alta atividade motora, podendo mesmo haver uma facilitação da aprendizagem no caso de a resposta requerer baixa atividade para ser emitida. Ao contrário, a hipótese do desamparo - que postula que a variável crítica é a aprendizagem de que os eventos do meio não são passíveis de controle - permite prever que o grau de atividade motora exigida para emissão da resposta no teste é irrelevante para a ocorrência do déficit de aprendizagem. Portanto, os sujeitos expostos aos choques incontroláveis deveriam apresentar o desamparo independentemente de a resposta de teste envolver alta ou baixa atividade motora. Nesse estudo, foram utilizadas duas respostas de fuga diferentes: "saltar" e "focinhar" (colocar o focinho em um orifício na parede). Considerou-se que e resposta de focinhar poderia ser classificada como resposta de baixa atividade motora uma vez que, para sua emissão, é indispensável que o sujeito esteja parado, com o corpo voltado para o orifício da parede e o focinho na altura do mesmo, sendo a única movimentação requerida a de esticar o pescoço, de forma a introduzir o focinho no orifício. Ao contrário, a resposta de saltar poderia ser classificada como de alta atividade motora, pois sua emissão requer o deslocamento global do sujeito que deve sair de um compartimento e chegar ao outro, do lado oposto da caixa. Portanto, o reforçamento negativo dessas duas respostas seleciona padrões opostos de atividade motora. Os resultados do primeiro estudo mostraram igual nível de desamparo nos testes com as respostas de saltar e de focinhar, confirmando a previsão da hipótese do desamparo e contrariando o previsto pela hipótese motora. No segundo experimento, foram testadas as previsões sobre o efeito de "imunização".
Segundo a hipótese motora, o efeito de imunização dependeria da combinação entre o grau de atividade motora exigido pelas respostas da primeira e última sessão, considerando as sessões de pré-treino/incontrolabilidade/teste. Se a resposta de fuga do pré-treino requeresse baixa atividade motora e a resposta de fuga no teste envolvesse alta movimentação corporal (combinação baixa/alta), o previsto seria um efeito de desamparo aprendido muito acentuado, contrário ao efeito de imunização. Na combinação oposta (alta/baixa), o previsto seria imunização acentuada (ou seja, aprendizagem de fuga a despeito da exposição prévia aos choques incontroláveis). $\mathrm{Na}$ previsão da hipótese do desamparo aprendido, a imunização se daria independente dessa combinação, pois o sujeito teria aprendido na primeira sessão que ele tinha controle sobre os choques, aprendizagem essa que o "imunizaria" contra a aprendizagem oposta da incontrolabilidade na sessão de tratamento. Portanto, apenas na combinação baixa/alta as previsões diferiam entre essas hipóteses. Utilizando, portanto, essa combinação (com as respostas de focinhar/saltar), os resultados mostraram acentuada imunização, confirmando a previsão da hipótese do desamparo aprendido.

Outro experimento confrontou ambas as hipóteses, sendo sua lógica baseada na equação que define a incontrolabilidade: $\mathrm{p}[\mathrm{S} / \mathrm{R}]=\mathrm{p}[\mathrm{S} / \mathrm{nR}]$, onde a probabilidade $(\mathrm{p})$ do estímulo (S) ocorrer após uma resposta $(\mathrm{R})$ é igual à sua probabilidade de ocorrer na ausência dessa resposta (nR). Hunziker (1981) manipulou a ocorrência de $\mathrm{R}$ ou de $\mathrm{nR}$ durante os choques incontroláveis, de forma que o término do choque (S) seguiria uma ou outra, operacionalizando a equação acima e evitando as supostas contingências acidentais. Para fazer esse teste, foi construída uma roda-de-atividade bastante estreita, que só permitia ao sujeito a opção de caminhar (R) ou ficar parado $(\mathrm{nR})$. Acoplada à roda havia um motor que poderia girá-la, travá-la ou deixá-la livre. Quando o motor girava a roda, o rato era forçado a caminhar $(\mathrm{R})$, e quando a travava o rato era forçado a ficar parado (nR). Na situação de roda-livre, os movimentos da roda eram determinados pelo sujeito: a emissão de $\mathrm{R}$ faria a roda girar e com $\mathrm{nR}$ a roda ficaria imóvel. Considerando como unidade de $\mathrm{R}$ cada $1 / 4$ de giro da roda, como $n R$ a ausência de movimentação da roda (menos que $1 / 4$ de giro) e o término do choque como o $\mathrm{S}$ em estudo, esse equipamento permitia a manipulação de $p(S / R)$ e de $p(S / n R)$. O experimento contou com seis grupos de ratos, sendo quatro expostos a choques incontroláveis e dois colocados na roda, sem choques. Dentre os animais que receberam choques, nenhum tinha controle sobre esses estímulos. Contudo, eles variaram entre si quanto ao arranjo de ambas as probabilidades dado o movimento da roda (girando/travada) durante os choques: grupo $100 \%(1,0 / 0,0)$, grupo $0 \%(0,0 / 1,0)$, grupo $50 \%(0,5 / 0,5)$ e grupo roda-livre. Após esse tratamento, todos os sujeitos foram igualmente expostos a uma contingência de reforçamento negativo (fuga) para a resposta de saltar. As hipóteses tinham previsões opostas quanto à essa aprendizagem de fuga apenas para os grupos $50 \%$ e $0 \%$ : se o processo fosse de inatividade aprendida, o esperado seria aprendizagem de fuga no grupo $50 \%$ e desamparo acentuado no grupo $0 \%$, invertendo-se essas previsões pela hipótese do desamparo aprendido. Ambas permitiam prever aprendizagem de fuga no grupo $100 \%$ e desamparo no grupo roda-livre. Os resultados mostraram desamparo apenas nos grupos $50 \%$ 
e roda-livre, sendo que os demais grupos aprenderam fuga, independentemente de terem ou não sido previamente expostos aos choques. Tais resultados confirmaram as previsões da hipótese do desamparo aprendido, além de terem validado o procedimento convencional (equivalente ao de roda-livre) como apropriado para o estudo da incontrolabilidade, definida pela equação descrita.

\section{Evolução das pesquisas sobre desamparo com animais}

Ao longo de quase quatro décadas de pesquisas, formou-se um conjunto considerável de informações sobre o desamparo aprendido. Alguns dos aspectos investigados apresentam resultados bastante consistentes entre si, mas outros têm resultados ainda controversos.

Dentre os aspectos bem estabelecidos está a generalidade entre espécies: além de obtido com sujeitos humanos, o desamparo aprendido vem sendo obtido com diferentes espécies de sujeitos não-humanos, tais como cães, gatos, ratos, camundongos, pombos, peixes, baratas (ver revisão em Peterson \& cols., 1993). Apesar dessa ampla generalidade entre espécies, é importante ressaltar que a maior parte das pesquisas utilizou ratos como sujeitos.

Também são bem estabelecidas as alterações fisiológicas produzidas pela incontrolabilidade dos choques. Além das alterações nos níveis centrais de alguns neurotransmissores (Weiss, Stone \& Harwell, 1970), estudos relataram que a exposição a choques incontroláveis pode também alterar a temperatura corporal (Endo \& Shikari, 2000), produzir aceleração no desenvolvimento de tumores e rebaixamento geral do sistema imunológico (Bem-Eliyahu, Yirma, Liebeskind, Taylor \& Gale, 1991; Laudenslager, Ryan, Drugan, Hyson \& Maier, 1983; Mormede, Dantzer, Michaud, Kelley \& Moal, 1988).

A incontrolabilidade dos choques tem sido apontada como a variável independente crítica nesses estudos (Peterson \& cols. 1993), embora a imprevisibilidade do estímulo também tenha sido sugerida como possivelmente relacionada a ele (Overmier, 1985; Overmier \& LoLordo, 1998). Contudo, os dados experimentais não têm confirmado essa última hipótese (por exemplo, Castelli, 2004). De uma maneira geral, a análise da (im)previsibilidade dos choques tem sido pouco considerada na grande maioria dos trabalhos publicados sobre o desamparo até o momento.

Características do estímulo manipulado também já foram identificadas como críticas na produção do desamparo em animais. A maioria dos estudos se restringe ao uso de choques elétricos que, como em outros processos controlados aversivamente (Fantino, 1973), têm como propriedades críticas sua intensidade, freqüência, duração e densidade (Altenor, Volpicelli \& Seligamn, 1979; Crowell \& Anderson, 1981; Crowell, Lupo, Cunninghan \& Anderson, 1978; Glazer \& Weiss, 1976ab; Lawry \& cols., 1978; Rosellini \& Seligman, 1978). Poucas variações do estímulo aversivo foram feitas, todas em estudos com humanos que utilizaram sons estridentes ou solução de anagramas (Hiroto \& Seligman, 1975; Matute, 1994).

Afora as características do estímulo, diversos estudos vêm mostrando sistematicamente que o desamparo também pode depender da contingência utilizada na sessão de teste.
Ao contrário dos cães utilizados nos primeiros estudos, ratos não apresentaram o desamparo quando testados em uma contingência de fuga que exigia deles correrem para o compartimento oposto ao que estavam (Maier, Albin \& Testa, 1973). O mesmo se verificou se deles era exigida a emissão de uma resposta de pressão à barra (Seligman \& Beagley, 1975). Contudo, quando se exigiram duas respostas de correr (razão fixa ou FR 2) ou três de pressão à barra (FR 3), os estudos relataram desamparo com ratos (Maier \& cols., 1973; Seligman \& Beagley, 1975). Após essas demonstrações, a contingência de reforçamento de fuga em FR2 para a resposta de correr passou a ser o procedimento de teste padrão para estudo do desamparo em ratos (Peterson \& cols., 1993). Contudo, essas contingências produzem resultados bastante inconsistentes que podem comprometer a análise do desamparo em ratos (Hunziker, 1977). Estudos recentes demonstraram que a importância da escolha da resposta de teste não é uma mera questão de aumentar a contingência de razão para reforçamento, mas sim de estabelecer claramente o seu controle pelas consequiências. Contingências que não geram padrão de aprendizagem de fuga nos animais ingênuos (como se verifica em muitos trabalhos publicados), não são adequadas para o estudo do desamparo aprendido (Hunziker, 2003). A suposta ausência do desamparo (quando são utilizadas contingências simples que exigem uma única resposta), não se confirmou, quando foram tomadas medidas para garantir o seu controle pelas consequiências: o desamparo tem sido observado em animais utilizando-se uma única resposta no teste de fuga, quer seja essa resposta saltar ou focinhar (Hunziker, 1981; 2003; Mestre \& Hunziker, 1996; Yano \& Hunziker, 2000).

Portanto, está amplamente demonstrado que a experiência com a incontrolabilidade do meio produz mudanças no organismo, da mesma maneira que algumas alterações do organismo produzem diferenças na forma como o sujeito lida com a incontrolabilidade do meio. Quer por identificar os substratos fisiológicos do comportamento, quer por identificar os contextos ambientais que modificam o organismo em diferentes níveis, os estudos sobre os efeitos da incontrolabilidade que envolvem variáveis ambientais e fisiológicas têm permitido que se amplie não somente a compreensão do desamparo aprendido, mas também do comportamento como um todo. Esse tipo de pesquisa oferece sustentação empírica para a asserção polêmica de Skinner de que "o comportamento de um organismo é simplesmente a fisiologia de uma anatomia" (1966, p. 1.205).

\section{Aspectos controversos no estudo do desamparo}

Apesar da replicação do desamparo em diferentes laboratórios, com numerosas espécies, há determinados resultados que são divergentes. Um deles diz respeito à replicabilidade entre sujeitos: enquanto Maier e Seligman (1976) relataram que cerca de um terço dos animais expostos a choques incontroláveis não apresentam o desamparo, há estudos que obtiveram desamparo com todos os ratos expostos a esse tratamento (Mestre \& Hunziker, 1996; Yano \& Hunziker, 2000).

Outro aspecto conflitante diz respeito à relevância do gênero do sujeito. $\mathrm{O}$ dado mais difundido é que o desamparo 
aprendido não é obtido com fêmeas de ratos ou camundongos (Calderone, George, Zachariou \& Picciotto, 2000; Steenberger, Heinsbroek, Van Haaren \& Van de Poll, 1989; 1990), embora diversos estudos tenham demonstrado desamparo equivalente com ratos machos ou fêmeas (Gouveia Jr, 2001; Hunziker \& Damiani, 1992; Yano \& Hunziker, 2000).

A generalidade do desamparo para contextos apetitivos também é um fator de discordância. Estímulos apetitivos, tais como alimento ou água, ministrados no tratamento e/ou no teste, foram utilizados em algumas pesquisas, com resultados contraditórios (Calef \& cols., 1984; Capelari \& Hunziker, 2005; Enberg, Welker, Hansen \& Thomaz, 1972; Ferrandiz \& Vincent, 1997; Job, 1988, 1989; Oakes, Rosenblum \& Fox, 1982; Rosellini, 1978). Ou seja, até o momento, não se pode afirmar se o desamparo ocorre em contextos que envolvam estímulos que não sejam aversivos.

Os efeitos de drogas antidepressivas (principalmente, da imipramina) têm sido descritos de forma diversa: alguns estudos com imipramina indicam que o desamparo só desaparece após tratamento crônico, mas não após uma única administração da droga (Petty \& Sherman, 1979; Sherman \& cols., 1982); contudo, resultados contrários foram descritos, mostrando ausência de desamparo em animais previamente tratados com choques incontroláveis que receberam uma única injeção de imipramina (Graeff \& Hunziker, 1988; Gouveia Jr, 2001; Hunziker, Buonomano \& Moura, 1986).

Quanto ao envolvimento do sistema opióide no desamparo, foi descrito que a hipoalgesia só se verificava quando o teste era realizado após a re-exposição dos animais a choques (Jackson, Maier \& Coon, 1979; Maier, Drugan \& Grau, 1982). Contudo, Hunziker (1992) demonstrou hipoalgesia opióide decorrente dos choques incontroláveis, sem essa re-exposição aos choques.

Por fim, são também discordantes os resultados relativos aos efeitos da liberação de um estímulo neutro após término dos choques incontroláveis: enquanto alguns estudos apontam que esse procedimento impede a ocorrência do desamparo, produzindo o chamado "efeito feedback" (Jackson \& Minor, 1988; Maier, Jackson \& Tomie, 1987; Volpicelli, Ulm \& Altenor, 1984), outros descrevem que esse tratamento foi inócuo, uma vez que o desamparo se deu apesar dos estímulos pós-choque (Damiani \& Hunziker, 1996).

Alguns desses resultados contraditórios podem ser decorrentes de uma base genética que determine diferentes sensibilidades ao tratamento com choques incontroláveis, conforme sugerido recentemente (Caldarone \& cols., 2000). Contudo é também provável que essas diferenças se devam, ao menos em parte, a questões metodológicas que têm sido negligenciadas em diversos estudos, que se apresentam com imprecisões no delineamento experimental (Hunziker, 2003).

\section{O desamparo aprendido como modelo animal de depressão}

A proposição do desamparo como um modelo animal de depressão (Seligman, 1975) foi motivada por algumas similaridades entre o comportamento de pessoas deprimidas e o comportamento dos animais que passavam pelos choques incontroláveis. Essas similaridades foram apontadas na sintomatologia, etiologia, prevenção e cura da depressão/ desampro. Por exemplo, a passividade dos animais frente aos choques, no teste, poderia se assemelhar à passividade do indivíduo deprimido, que não atua sobre o seu meio. Quanto à etiologia, sabe-se que eventos traumáticos, fora do controle do sujeito, podem ser desencadeantes de alguns tipos de depressão humana, bem como o são no desenvolvimento do desamparo em animais (Willner, 1984; 1985). Além disso, alterações bioquímicas encontradas em pacientes depressivos foram também encontradas em animais que apresentaram o desamparo aprendido (Willner, 1991). Por sua vez, a prevenção e o tratamento clínico poderiam ter um paralelo comportamental nos estudos sobre "imunização" e "reversão", além do paralelo da farmacoterapia, dado que drogas que têm comprovado efeito antidepressivo em sujeitos humanos também impedem o aparecimento do desamparo em animais (Petty \& Sherman, 1979). Esses resultados têm justificado o uso do desamparo, em pesquisas com animais, para identificação de novos fármacos com potencial antidepressivo em humanos (Willner, 1985;1991).

Essa suposta representatividade da depressão humana deu um grande impulso na investigação do desamparo, estabelecendo uma atrativa ponte entre o laboratório e a clínica (Maier \& Seligman, 1993; Peterson \& cols., 1993; Willner, 1984, 1991). Além do aspecto de farmacoterapia, a análise do comportamento depressivo também tem sido atendida com esses estudos. Por exemplo, um aspecto crítico do desamparo é a redução da sensibilidade às contingências de reforçamento em vigor, ou seja, a alteração de um dos mecanismos considerados básicos na adaptação dos indivíduos ao seu ambiente (Skinner, 1981). Assim, a compreensão de um processo que enfraquece a possibilidade de seleção pelas consequiências assume importância crítica para a compreensão do comportamento, especialmente daqueles considerados patológicos, que são sinônimos de má adaptação do indivíduo. Funcionalmente, se considerarmos que a pessoa deprimida sofre de falta de reforçadores (Ferster, 1973), podemos dizer que o desamparo, por reduzir a sensibilidade ao reforçamento, pode ser potencialmente um bom modelo animal de depressão. No laboratório, essa perda da função reforçadora pode ser indicada pela não alteração da probabilidade da resposta de fuga, apresentada no teste por alguns sujeitos, conforme já relatado. Na depressão humana, a falta de reforçadores pode ser analisada pelo "desinteresse", muitas vezes verbalizado pelos indivíduos, relativo a aspectos do seu ambiente que já foram reforçadores, ou mesmo pela falta da função reforçadora de alguns estímulos, que, embora ocorram contingentes às respostas, não as mantém.

Apesar de muito estimulante, essa associação entre um efeito produzido no laboratório com aqueles vistos na clínica deve ser olhada com cautela para que se evitem análises precipitadas ou mesmo inadequadas. Conforme analisado por Hunziker e Perez-Acosta (2001), as analogias entre laboratório e clínica só se justificam se fundamentadas em rigorosa análise do comportamento. Tanto o desamparo animal, como a depressão humana, não podem ser definidos com base em mera topografia de respostas, mas sim pelas relações funcionais que envolvem. Caso essas relações não sejam analisadas (e muitas vezes não o são), o uso desse ou de qualquer modelo animal, como análogo a uma 
psicopatologia, se transforma em aplicação simplista, que não produz avanço do conhecimento.

\section{Perspectivas de investigação}

De uma maneira geral, a perda do rigor metodológico, aliada a diversas interpretações cognitivistas do efeito, afastou os analistas do comportamento do estudo do desamparo (Hunziker, 1996). Isso pode ser constatado na ausência de trabalhos sobre o tema nos periódicos mais rigorosos da área, tal como o Journal of the Experimental Analysis of Behavior. A conseqüência dessa exclusão foi que o desamparo passou a ser investigado por pesquisadores não interessados nas questões teóricas e metodológicas envolvidas no comportamento em estudo. Com isso, perdemos todos: por um lado, os analistas do comportamento, que deixaram de lado um delineamento experimental rico para o estudo do comportamento, e, por outro, a comunidade científica, repleta de estudos mal conduzidos metodologicamente que criaram a ilusão de um fenômeno muito estudado, mas que é, de fato, pouco conhecido.

Há ainda muito para ser investigado sobre o desamparo aprendido, com procedimentos confiáveis e generalizações cuidadosas. Sua vinculação com a depressão humana torna, sem dúvida, bastante desafiante o estudo do desamparo. Porém, as análises precisam ser sólidas, baseadas em dados experimentais. Por exemplo, é crítico que se tenha mais bem estabelecida a relação entre os contextos aversivo e apetitivo, tanto na produção do desamparo como da sua imunização. Esses dados podem trazer informações que ajudem na compreensão da etiologia, tratamento e prevenção do desamparo e, talvez, da depressão humana. Será esse efeito/patologia determinado apenas por contextos aversivos? A sua reversão pode se dar com tratamentos baseados em reforçamento positivo? É também relevante que se explore como o repertório comportamental prévio interfere na sensibilidade ao desenvolvimento do desamparo. Será que histórias de reforçamento que geram diferentes padrões comportamentais (tais como, variabilidade ou repetição) podem influenciar diferencialmente o futuro desenvolvimento do desamparo? Outro fator crítico é o grau de sociabilidade dos sujeitos: indivíduos criados em grupo ou isoladamente reagem igualmente a eventos aversivos incontroláveis? Seu status social no grupo de convivência (dominante ou submisso), pode ser um fator que influencia no desenvolvimento do desamparo? Enfim, perguntas não faltam. O que essa área precisa é de estudos desenvolvidos com rigor metodológico e conceitual. Sem isso, a relação do desamparo com a depressão clínica torna-se mera especulação. Cabe aos analistas do comportamento assumir essa empreitada.

\section{Referências}

Altenor, A., Volpicelli, J. R. \& Seligman, M. E. P. (1979). Debilitated shock escape is produced by short- and long-duration inescapable shock: Learned helplessness vs. learned inactivity. Bulletin of Psychonomic Society, 14(3), 337-339.

Bem-Eliyahu, S., Yirma, R., Liebeskind, J. C., Taylor, A. N. \& Gale, R. P. (1991). Stress increases metastatic spread of a mammary tumor in rats: Evidence for mediation by immune system. Brain, Behavior, and Immunity, 5(2), 193-206.
Bracewell, J. R. \& Black, A. H. (1974). The effects of restraint and noncontingent preshock on subsequent escape learning in the rat. Learning and Motivation, 5, 53-69.

Calderone, B. J, George, T. P., Zachariou, V., \& Picciotto, M. R. (2000). Gender differences in learned helplessness behavior are influenced by genetic background. Pharmacology, Biochemistry and Behavior, 66(4), 811-817.

Calef, R. S., Metz, R. A., Atkinson, T. L., Pellerzi, R. C., Taylor, K. S. \& Geller, E. S. (1984). Acquisition of running in straight alley following experience with response - independent food. Bulletin of the Psychonomic Society, 22(11), 67-69.

Capelari, A. \& Hunziker, M.H.L. (2005). Aprendizagem de fuga após estímulos apetitivos incontroláveis. Psicologia: Teoria e Pesquisa, 21(1), 99-107.

Castelli, M. C. Z. (2004). Efeitos da previsibilidade de estímulos no desamparo aprendido: uma comparação entre ratos machos e fêmeas. Tese de Doutorado, Universidade de São Paulo, São Paulo.

Catania, A. C. (1998). Learning. Englewood Cliffs, N.J.: PrenticeHall.

Crowell, C. R. \& Anderson, D. C. (1981). Influence of duration and number of inescapable shocks on intra-shock activity and subsequent interference effects. Animal Learning and Behavior, 9, 28-37.

Crowell, C. R., Lupo, J. V., Cunninghan, C. L. \& Anderson, D. C. (1978). Temporal form of hock is a determinant of magnitude of interference with escape-response learning produced by exposure in the inescapable shock. Bulletin of the Psychonomic Society, 12(5), 401-410.

Damiani, K. \& Hunziker, M. H. L. (1996). Effects of S-S associations on learned helplessness: A parametric study in female and male rats [Resumo]. Em Association for Behavior Analysis (Org.). 22thnd Annual Convention, Program (p. 60). San Francisco: ABA.

Enberg, L. A., Welker. R. L., Hansen, G. \& Thomas, D. R. (1972). Acquisition of key pecking via auto shaping as a function to prior experience: "Learned Laziness"? Science, 178, 10021004.

Endo, Y. \& Shikari, K. (2000). Behavior and body temperature in rats following chronic foot shock or psychological stress exposure. Physiology and Behavior, 71(3), 263-268.

Erbetta, K. C. H. S. (2004). Efeito do tratamento com reforço positivo sobre o desamparo aprendido em ratos. Dissertação de Mestrado, Universidade de São Paulo, São Paulo.

Fantino, E. (1973). Aversive control. Em J. A. Nevin (Org.), The study of behavior (pp. 239-279). Glenview, Illinois: Scott, Foresman and Company.

Ferrandiz, P. \& Vincent, F. (1997). The conditioned attention theory and bifactorial theory on the learned helplessness syndrome in appetitive contexts. International Journal of Psychology, 32(6), 399-408.

Glazer, H. I. \& Weiss, J. M. (1976a). Long term and transitory interference effects. Journal of Experimental Psychology: Animal Behavior Processes, 2, 191-201.

Glazer, H. I. \& Weiss, J. M. (1976b). Long-term interference effect: An alternative to learned helplessness. Journal of Experimental Psychology: Animal Behavior Processes, 2, 202-213.

Gouveia Jr., A. (2001). Efeitos da administração aguda de imipramina sobre o desamparo aprendido em ratos machos e fêmeas. Acta Comportamentalia, 9(1), 19-29. 
Graeff, E. O. \& Hunziker, M. H. L. (1988). Sensibilidade do efeito de interferência de longa duração ao tratamento crônico e agudo com imipramina em ratos [Resumo]. Sociedade de Psicologia de Ribeirão Preto (Org.), XVIII Reunião Anual de Psicologia, Resumos (p. 217). Ribeirão Preto: SPRP.

Hiroto, D. S. \& Seligman, M. E. P. (1975). Generality of learned helplessness in man. Journal of Personality and Social Psychology, 31(4), 311-327.

Hunziker, M. H. L. (1977). Efeitos da exposição prévia a choques não contingentes sobre a aquisição do comportamento de fuga como função de algumas dimensões da resposta. Dissertação de Mestrado, Universidade de São Paulo, São Paulo.

Hunziker, M. H. L. (1981). Um estudo sobre a incontrolabilidade. Considerações metodológicas, uma análise experimental. Tese de Doutorado, Universidade de São Paulo, São Paulo.

Hunziker, M. H. L. (1992). Opioid nature of learned helplessness and stress-induced analgesia observed without re-exposure to shock. Behavioural Pharmacology, 3(2), 117-121.

Hunziker, M. H. L. (1996). Learned helplessness: An important phenomenon neglected in the behavior analysis literature [Resumo]. 3rd International Congress on Behaviorism and the Sciences of Behavior Program (p. 18). Okohama (Japão): BSC.

Hunziker, M. H. L. (2003). Desamparo aprendido. Tese de LivreDocência, Universidade de São Paulo, São Paulo.

Hunziker, M. H. L., Buonomano, D. V. \& Moura, M. D. (1986). Efeito da administração aguda de imipramina sobre o comportamento de ratos [Resumo]. Em Sociedade de Psicologia de Ribeirão Preto (Org.), Resumos de Comunicações Científicas, XVI Reunião Anual de Psicologia (p. 35). Ribeirão Preto: SPRP.

Hunziker, M. H. L. \& Damiani, K. (1992). Non-contingent aversive stimuli and escape learning in rats: a male/female comparison [Resumo]. Association for Behavior Analysis (Org.), 18th Annual Convention, Program (p. 101). San Francisco: ABA.

Hunziker, M. H. L. \& Perez-Acosta, A. M. (2001). Modelos animales en psicopatología: ¿Una contribución o una ilusión? Avances en Psicología Clínica Latinoamericana, 19, 37-50.

Jackson, R. L., Maier, S. F. \& Coon, D. J. (1979). Long-term analgesic effects of inescapable shock and learned helplessness. Science, 206, 91-94.

Jackson, R. L. \& Minor, T. R. (1988). Effects of signaling inescapable shock on subsequent escape learning: Implications for theories of coping and "learned helplessness". Journal of Experimental Psychology: Animal Behavior Process, 14, 390-400.

Job, R. F. S. (1988). Interference and facilitation produced by noncontingent reinforcement in appetitive situation. Animal Learning and Behavior, 16, 451-460.

Job, R. F. S (1989). A test of proposed mechanism underlying the interference effect produced by contingent food presentation. Learning and Motivation, 20(2), 153-177.

Laudenslager, M. L., Ryan, S. M., Drugan, R. C., Hyson, R. L. \& Maier, S. F. (1983). Coping and immunosupression: Inescapable but not escapable shock suppresses lymphocyte proliferation. Science, 221, 568-570.

Lawry, J. A., Lupo, V., Overmier, J. J., Kochevar, J., Hollis, K. L. \& Anderson, D. C. (1978). Interference with avoidance behavior as a function of qualitative properties of inescapable shocks. Animal Learning and Behavior, 6, 147-154.

Levis, D. J. (1976). Learned helplessness: A reply and alternative SR interpretation. Journal of Experimental Psychology: General, 105(1), 47-65.
Maier, S. F.; Albin, R. W. \& Testa, T. J. (1973). Failure to learn to escape in rats previously exposed to inescapable shock depends on nature of escape response. Journal of Comparative and Physiological Psychology, 85(3), 581-592.

Maier, S. F., Drugan, R. C. \& Grau, J. W. (1982). Controllability, coping behavior, and stress induced analgesia in the rat. Pain, 12, 47-56.

Maier, S. F., Jackson, R. L. \& Tomie, A. (1987). Potentiation, overshadowing, and prior exposure to inescapable shock. Journal of the Experimental Psychology: Animal Behavior Process, 13(3), 260-270.

Maier, S. F. \& Seligman, M. E. P. (1976). Learned helplessness: Theory and evidence. Journal of Experimental Psychology: General, 105(1), 3-46.

Maier, S. F.; Seligman M. E. P. \& Solomon, R. L. (1969). Pavlovian fear conditioning and learned helplessness: Effects on escape and avoidance behavior of (a) the CS-US contingency and (b) the independence of the US and voluntary responding. Em B. A. Campbell \& K. M. Church (Orgs.), Punishment and aversive behavior. New York: Appleton-Century-Crofts.

Mestre, M. B. A. \& Hunziker, M. H. L. (1996) O desamparo aprendido, em ratos adultos, como função de experiências aversivas incontroláveis na infância. Tuiuti: Ciência e Cultura, 6(2), 25-47.

Mormede, P., Dantzer, R., Michaud, B., Kelley, K. W. \& Moal, M. L. (1988). Influence of stressor predictability and behavioral control on lymphocyte reactivity, antibody responses and neuroendocrine activation in rats. Physiology and Behavior, 43(6), 577-583.

Mowrer, O. H. (1947). On the dual nature learning - A reinterpretation of "conditioning" and "problem-solving". Harvard Educational Review, 17(2), 102-150.

Oakes, W. F.; Rosenblum, J. L. \& Fox, P. E. (1982). "Manna from haven": The effect of noncontingent appetitive reinforces on learning in rats Bulletin of the Psychonomic Society, 19(2), 123-126.

Overmier, J. B. (1985). Toward a reanalysis of the causal structure of the learned helplessness syndrome. Em F. R. Brush \& J. B. Overmier (Orgs.), Affect, conditioning and cognition: Essays on the determinants of behavior (pp. 211-227). Hillsdale, NJ: Erlbaum.

Overmier, J. B. \& Leaf, R. C. (1965). Effects of discriminative Pavlovian fear conditioning upon previously or subsequently acquired avoidance responding. Journal of Comparative and Physiological Psychology, 60(3), 213-218.

Overmier, J. B. \& LoLordo, V. M. (1998). Learned helplessness. Em W. O'Donohue (Org.), Learning and Behavior Therapy (pp. 134-168). Boston: Allyn and Bacon.

Overmier J. B. \& Seligman, M. E. P. (1967). Effects of inescapable shock upon subsequent escape and avoidance learning. Journal of Comparative and Physiological Psychology, 63(1), 23-33.

Peterson, C., Maier, S. F. \& Seligman, M. E. P. (1993). Learned helplessness: a theory for the age of personal control. New York: Oxford University Press.

Petty, F. \& Sherman, A. D. (1979). Reversal of learned helplessness by imipramine. Communications in Psychopharmacology, 3(3), 371-373.

Rescorla, R. \& Solomon, R. L. (1967). Two processes learning theory: Relationships between Pavlovian conditioning and instrumental learning. Psychological Review, 74(3), 151-182. 
Rosellini, R. A. (1978). Inescapable shock interferes with acquisition of an appetitive operant. Animal Learning and Behavior, 6(2), 155-159.

Rosellini, R. A. \& Seligman, M. E. P. (1978). Role of shock intensity in the learned helplessness paradigm. Animal Learning and Behavior, 6(2), 143-146.

Seligman, M. E. P. (1975). Helplessness: On depression, development and death. San Francisco: Freeman.

Seligman, M. E. P. \& Beagley, G. (1975). Learned helplessness in the rat. Journal of Comparative and Physiological Psychology, 88(6), 534-541.

Seligman, M. E. P. \& Maier, S. F. (1967). Failure to escape traumatic shock. Journal of Experimental Psychology, 74, 1-9.

Skinner, B. F. (1966). The phylogeny and ontogeny of behavior. Science, 153(12), 1204-1213.

Skinner, B. F. (1974). About behaviorism. New York: Knopf.

Skinner, B. F. (1981). Selection by consequences. Science, 213(6), 501-504.

Steenberger, H. L., Heinsbroek, R. P.W., Van Haaren, F. \& van de Poll, N.E. (1989). Sex-dependent effects of inescapable shock administration on behavior and subsequent escape performance in rats. Physiology and Behavior, 45, 781-787.
Volpicelli, J. R., Ulm, R. R., Altenor, A. (1984). Feedback during exposure to inescapable shocks and subsequent shock-escape performance. Learning and Motivation, 15(3), 279-286.

Weiss, J. M., Stone, E. A. \& Harwell, N. (1970). Coping behavior and brain norepinefrine level in rats. Journal of Comparative and Physiological Psychology, 72(2), 153-160.

Willner, P. (1984). The validity of animal models of depression. Psychopharmacology, 3(1), 1-16.

Willner, P. (1985). Depression: A psychobiological synthesis. New York: Wiley.

Willner, P. (1991). Models of depression. Em P. Willner (Org.), Behavioural models in psychopharmacology: Theoretical, industrial, and clinical perspectives (pp. 89-125). Cambridge: Cambridge University Press.

Yano, Y. \& Hunziker, M. H. L. (2000). Desamparo Aprendido e imunização com diferentes respostas de fuga. Acta Comportamentalia, 8(2), 143-166.

Recebido em 28.06.2004 Primeira decisão editorial em 31.01.2005

Versão final em 03.03.2005 Aceito em 08.08.2005 